\title{
THE SOURCE OF THE SURFACE FLUORESCENCE OF NANODIAMONDS
}

\author{
${ }^{1}$ Alexey VERVALD, ${ }^{2}$ Oleg KUDRYAVTSEV, ${ }^{1}$ Andrey LACHKO, ${ }^{1}$ Sergey BURIKOV, \\ ${ }^{3}$ Olga SHENDEROVA, ${ }^{2}$ Igor VLASOV, ${ }^{1}$ Tatiana DOLENKO
}

\author{
${ }^{1}$ M.V. Lomonosov Moscow State University, Faculty of Physics, Moscow, Russian Federation, \\ alexey.vervald@physics.msu.ru \\ ${ }^{2}$ General Physics Institute, Russian Academy of Sciences, Moscow, Russian Federation \\ ${ }^{3}$ Adámas Nanotechnologies, Inc., Raleigh, USA
}

https://doi.org/10.37904/nanocon.2019.8569

\begin{abstract}
In this paper photoluminescence properties of aqueous suspensions of carbon nanoparticles (nanodiamonds, carbon dots and complexes on their basis) with different ratio of surface carbon in $\mathrm{sp}^{3}$ - and $\mathrm{sp}^{2}$-hybridizations were studied by methods of Raman, fluorescence and IR absorption spectroscopy. It was found that intensity of photoluminescence correlates with amount of carbon in non-diamond phase on the surface of nanoparticles. Nanoparticles with high non-diamond carbon phase content revealed more intense photoluminescence. The obtained results support the hypothesis about surface nature of photoluminescence of nanodiamonds and carbon dots.
\end{abstract}

Keywords: Carbon nanoparticles, photoluminescence, carbon hybridization, spectroscopy

\section{INTRODUCTION}

Currently, a development of photoluminescent biomarkers - non-toxic, biocompatible, stable luminescent nanomaterials is extremely relevant. Nanodiamonds are one of the most promising [1-4] materials. In addition to the mentioned above properties, they have sorption properties, which makes it possible to use them as adsorbents or drug carriers [5-7]. The unique photoluminescent properties made the metal-containing quantum dots competitive with organic dyes and determined the high potential of their use as biological markers [1,2]. However, detected toxicity of these quantum dots limits the field of their application. An alternative to metalcontaining quantum dots are carbon quantum dots, which have not only stable photoluminescence, but also high biocompatibility [3].

One of the most important discoveries of the last decades in the field of photoluminescent nanoparticles is the detection of bright luminescence in carbon nanoparticles (CNP) - carbon dots (CD) and nanodiamonds (ND) during passivation of their surface or irradiation of diamond nanocrystals by high-energy particles. These luminescent carbon nanoparticles gradually come to the leaders among high-contrast optical materials for biovisualization due to compliance to the criteria of applicability in biology and medicine [1-7]. However, for the proper use of such CNP as photoluminescent biomarkers and drug carriers, it is necessary to be able to govern their photoluminescent and sorption properties. And it is possible only on the basis of understanding mechanisms of formation of photoluminescence and sorption ability of CNP. Unfortunately, at the moment the nature of surface fluorescence of CNP remains unclear. Photoluminescence caused by defects in the surface of carbon in $\mathrm{sp}^{2}$-hybridization is considered as one of the possible mechanisms of photoluminescence of $C D$ and ND. Defects are defined as any disturbance of the carbon structure in $\mathrm{sp}^{2}$-hybridization which provides formation of additional surface electron levels or traps. According to assumption of the authors [8], the optical properties of carbon nanomaterials (including photoluminescent ones [9]) having carbon in their structure in both $\mathrm{sp}^{2}$-and $\mathrm{sp}^{3}$-hybridizations are determined by the $\pi$ states of electrons of carbon atoms with $\mathrm{sp}^{2}$ - 
hybridization. Photoluminescence in this system depends on electron-hole recombination in localized $\pi$ states in the band gap [10,11]. Due to the wide size distribution of such $\mathrm{sp}^{2}$ clusters, caused by low value of the binding energy, wide spectrum of photoluminescence is observed from the visible range to the near IR [9].

Thus, currently, one of the most common in the literature is the hypothesis that the photoluminescence of the CNP is to some extent determined by the amount of non-diamond phase of carbon, located on the surface of the CNP after cleaning and functionalization of the surface [9]. In this paper, this hypothesis is verified: the results of study of influence of the amount of non-diamond phase of carbon (primarily sp²-hybridized carbon) on the surface of nanoparticles on their photoluminescent properties are presented. For change and control of the amount of non-diamond carbon phase on the surface of nanoparticles, several types of CNP surface cleaning were carried out. Fluorescence spectroscopy, confocal Raman spectroscopy, and IR absorption spectroscopy were used to study the dependence of the CNP fluorescence on the amount of carbon in nondiamond phase on the surface of nanoparticlesach or solution.

\section{MATERIALS AND METHODS}

\subsection{Materials}

In this work, CNP, which significantly differ in the amount of non-diamond carbon phase on the surface: detonation nanodiamonds (DND), nanodiamonds, decorated with carbon dots (CDDND), and carbon dots (CD) themselves were investigated.

DND and CDDND were synthesized by the explosion of a mixture of trinitrotoluene (TNT) and 1,3,5 trinitrotoluene-1,3,5-triazine (hexogen) in a water-cooled medium [12]. Purification and surface treatment of nanoparticles were carried out in the International Technology Center (Raleigh, USA). CD are the product of chemical reactions of intercalation, delamination and oxidation of $400 \mathrm{~nm}$ nanographite sheets. CD synthesis was carried out in the international Technology Center [13].

In order to change and control the amount of carbon in non-diamond phase on the surface of CDDND, their surface was cleaned in perchloric acid and in the mixture of sulfuric and nitric acids. The CDDND sample was treated with perchloric acid $\mathrm{HClO}_{4}$ for 3 hours at $180^{\circ} \mathrm{C}$ and mixture of nitric and sulfuric acids $\mathrm{HNO}_{3}+\mathrm{H}_{2} \mathrm{SO}_{4}$ in $1: 3$ ratio for 3 hours at $130^{\circ} \mathrm{C}$. In order to remove the acids, the samples were centrifuged till deposition, followed by the removal of the supernatant and dilution of the precipitate with distilled water. The process was repeated 5 times. The final removal of acids was carried out by placing the samples in a vacuum chamber, bringing to crystallization and subsequent sublimation of the solvent. Below in the text, these two samples will be denoted as $\mathrm{CDDND}\left(\mathrm{HClO}_{4}\right)$ and $\mathrm{CDDND}\left(\mathrm{HNO}_{3}+\mathrm{H}_{2} \mathrm{SO}_{4}\right)$.

Deionized bi-distilled water with electrical conductivity of $0.1 \mu \mathrm{S} / \mathrm{cm}$ (Milli-Q) was used to prepare aqueous suspensions of the samples.

\subsection{Methods of research}

The composition of the samples was studied by Raman spectroscopy using the Horiba Jobin Yvon LabRAM $\mathrm{HR}-800$ confocal laser spectrometer, wavelength $473 \mathrm{~nm}$, the practical spectral resolution was $1 \mathrm{~cm}^{-1}$.

The study of the functional cover of the CNP was carried out using IR absorption spectroscopy using the Varian $640-$ IR FT-IR spectrometer, spectral resolution was $4 \mathrm{~cm}^{-1}$.

Photoluminescence spectra of the samples were obtained when excited by diode laser $\left(\lambda_{e x}=405 \mathrm{~nm}\right.$, output power - up to $200 \mathrm{~mW}$ ). PMT Hamamatsu H-8259-01, operating in the photon counting mode was used as detector. The PMT sensitivity in the visible range was 10 photons/count. 


\section{RESULTS AND DISCUSSION}

In this paper, the correlation between the intensity of fluorescence of CNP with different amounts of nondiamond phase of carbon on the surface and the fraction of this carbon in these nanoparticles. In total, 5 types of CNP were used: $\mathrm{CD}, \mathrm{ND}, \mathrm{CDDND}, \mathrm{CDDND}\left(\mathrm{HClO}_{4}\right)$ and $\mathrm{CDDND}\left(\mathrm{HNO}_{3}+\mathrm{H}_{2} \mathrm{SO}_{4}\right)$. The surface of nanoparticles was characterized by IR absorption spectroscopy and confocal Raman spectroscopy. The aqueous suspensions of $\mathrm{ND}, \mathrm{CDDND}, \mathrm{CDDND}\left(\mathrm{HClO}_{4}\right)$ and $\mathrm{CDDND}\left(\mathrm{HNO}_{3}+\mathrm{H}_{2} \mathrm{SO}_{4}\right)$ with concentrations $1 \mathrm{~g} / \mathrm{l}$ and suspensions of $C D$ with concentration $0.001 \mathrm{~g} / \mathrm{l}$ were studied. It was established with the help of method of dynamic light scattering that sizes of all samples were about $5 \mathrm{~nm}$.

\section{ANALYSIS OF SURFACE GROUPS OF CNP}

The method of IR absorption spectroscopy was used for determination of the composition of the surface groups of CNP. The IR absorption spectra of depositions of all 5 evaporated CNP samples are shown in Figure 1.

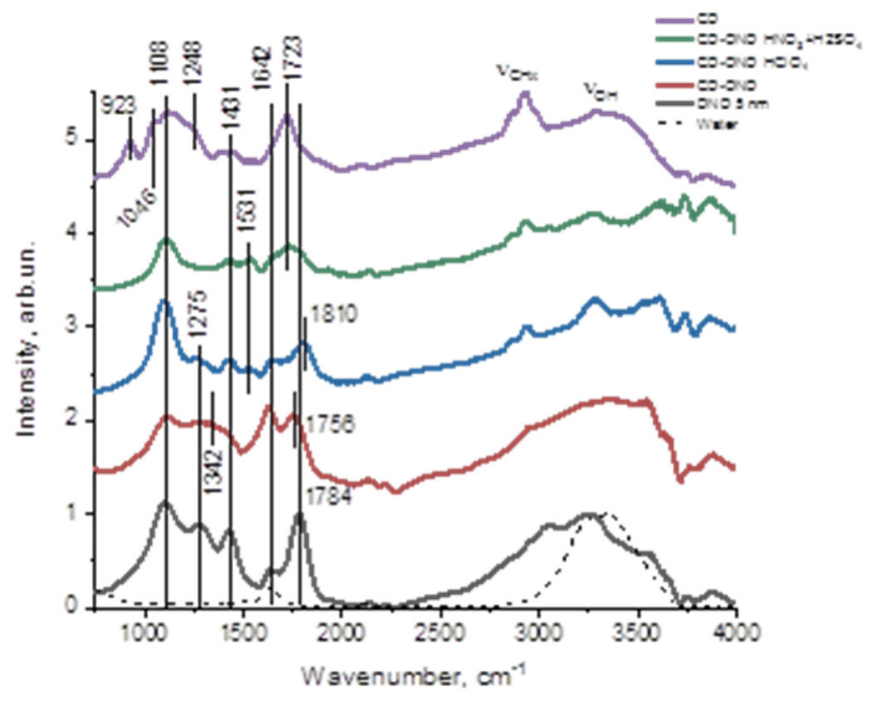

Figure $1 \mathrm{IR}$ absorption spectrum of studied CNP evaporated from water suspensions

In the spectra of IR absorption of all five samples one can observe a lot of bands corresponding to the vibrations of oxygen-containing functional surface groups. Near $1630 \mathrm{~cm}^{-1}$ there is a band of bending vibrations of $\mathrm{OH}$ groups. The intense band with a maximum, which varies for different samples in the range of $1720-1810 \mathrm{~cm}^{-1}$, corresponds to the valence vibrations of $\mathrm{C}=\mathrm{O}$ groups and indicates a high carboxylation of the nanoparticle surface. This is confirmed by the band of bending vibrations of groups $\mathrm{C}-\mathrm{O}-\mathrm{C}$ in the region of $1100-1140 \mathrm{~cm}^{-1}[14]$. Thus, it follows from the IR absorption spectroscopy data that carboxyl groups dominate on the surface of all samples. This means that the difference in the photoluminescent properties of the samples is not caused by the functional cover of CNP.

\section{DETERMINATION OF THE RATIO OF CARBON IN THE NON-DIAMOND PHASE AND WITH SP3 HYBRIDIZATION ON THE SURFACE OF CNP}

The ratio of carbon in non-diamond phase and with sp3 hybridization on the surface of the CNP was determined by confocal RAMAN spectroscopy. The broadband signal of CNP photoluminescence was approximated linear function and subtracted from the obtained Raman spectra. The obtained Raman spectra are presented in Figure 2. 


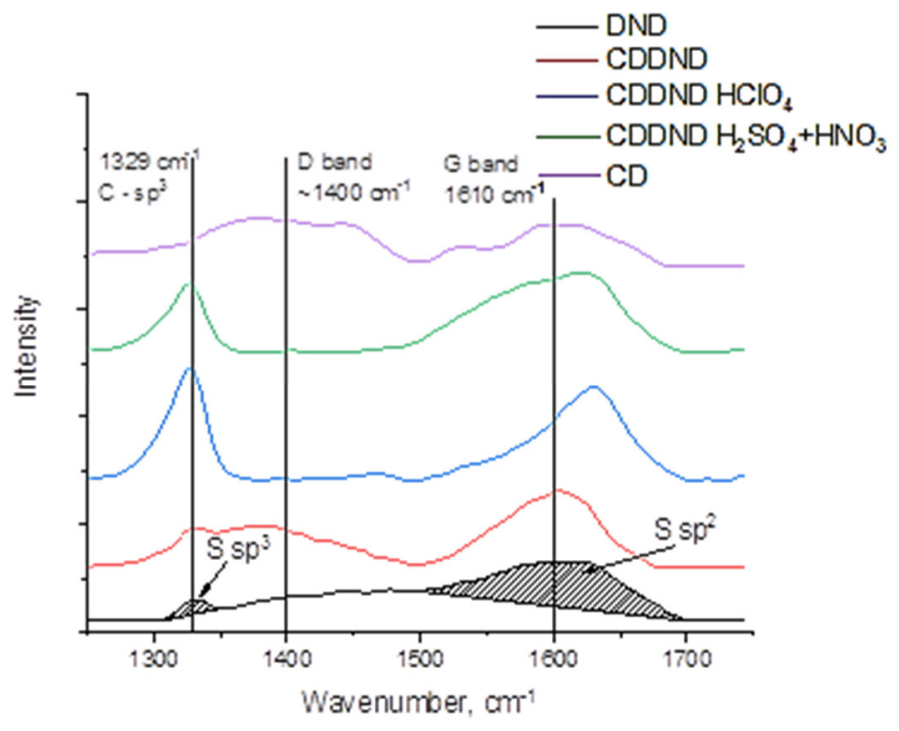

Figure 2 Raman spectra of powdered samples of all carbon nanoparticles

As it follows from the obtained data, in the spectra of all ND there are bands of carbon with $\mathrm{sp}^{3}$ hybridization with a maximum near $1329 \mathrm{~cm}^{-1}$. The shift of this band from the value of $1332 \mathrm{~cm}^{-1}$, corresponding to the position of this band in the bulk diamond, and its broadening are caused by the phonon confinement effect $[15,16]$ and indicate the small sizes of studied ND. In the spectra of all samples one can observe D- and Gbands with maximums near $1400 \mathrm{~cm}^{-1}$ and $1610 \mathrm{~cm}^{-1}$, respectively. Since the G-band overlaps with the water Raman bending band (with maximum near $1630 \mathrm{~cm}^{-1}$ ), in order to obtain useful G-signal, the band of bending vibrations of $\mathrm{OH}$ groups of water was subtracted from the band in the range from 1500 to $1700 \mathrm{~cm}^{-1}$. The integral intensity of band of the $\mathrm{sp}^{3}$-hybridized carbon with maximum near $1329 \mathrm{~cm}^{-1} \mathrm{~S}\left(\mathrm{sp}^{3}\right)$ is proportional to the amount of $\mathrm{sp}^{3}$-hybridized carbon in the samples. Since in the range from $1500 \mathrm{~cm}^{-1}$ to $1700 \mathrm{~cm}^{-1}$ the G-band of the $\mathrm{sp}^{2}$-hybridization carbon dominates, the $\mathrm{sp}^{2}$ designation will be used in the calculations. By calculating the integral intensities of these Raman bands, the ratio of amounts of carbon in different hybridizations $\left(\mathrm{V}\left(\mathrm{sp}^{2}\right)\right.$ and $\left.\mathrm{V}\left(\mathrm{sp}^{3}\right)\right)$ can be obtained by the following formula:

$\frac{V\left(s p^{2}\right)}{V\left(s p^{3}\right)}=\frac{s\left(s p^{2}\right)}{S\left(s p^{3}\right)} / \frac{\sigma\left(s p^{2}\right)}{\sigma\left(s p^{3}\right)^{\prime}}$

where:

$\sigma$ - cross-section of scattering of carbon in the corresponding hybridization. $\left(\mathrm{cm}^{2}\right)$

$S$ - integral intensity of band. (a. u.)

$\mathrm{V}$ - ratio of atoms in special hybridization. (a. u.)

The cross-section of Raman scattering for different bands of CNP spectra is different and also depends on the excitation wavelength. Unfortunately, for the excitation wavelength $473 \mathrm{~nm}$ used in this study, we could not find in literature the values of the scattering cross sections of CNP. The values of scattering cross-sections of diamond ( $\mathrm{sp}^{3}$-hybridization of carbon) $\sigma\left(\mathrm{sp}^{3}\right)=2.7 \cdot 10^{-29} \mathrm{~cm}^{2}$ and graphite $\sigma\left(\mathrm{sp}^{2}\right)=7 \cdot 10^{-28} \mathrm{~cm}^{2}$ under excitation by laser radiation with wavelength of $514.5 \mathrm{~nm}$ obtained by the authors [17] can be used as one of the most suitable for our conditions.

The results of calculations of the ratio of integral intensities of the corresponding spectral bands of Raman spectra and the amount of carbon in $\mathrm{sp}^{2}$ and $\mathrm{sp}^{3}$ hybridizations, as well as percent content of the amount of $\mathrm{sp}^{2}$-hybridized carbon from the total amount of carbon in the studied CNP are presented in Table 1. 
Table 1 The ratio of the integral intensities of spectral Raman bands and the amount of carbon $\mathrm{sp}^{2} / \mathrm{sp}^{3}$ hybridizations and percent content of carbon of non-diamond phase from the total carbon

\begin{tabular}{|l|l|l|l|l|l|}
\hline & DND & CDDND & CDDND $\mathrm{HCIO}_{4}$ & $\mathrm{CDDND}_{2} \mathrm{SO}_{4}+\mathrm{HNO}_{3}$ & $\mathrm{CD}$ \\
\hline $\mathrm{S}\left(\mathrm{sp}^{2}\right) / \mathrm{S}\left(\mathrm{sp}^{3}\right)$ & $14.2 \pm 2.4$ & $19.1 \pm 0.4$ & $1.62 \pm 0.10$ & $3.6 \pm 0.3$ & - \\
\hline $\mathrm{V}\left(\mathrm{sp}^{2}\right) / \mathrm{V}\left(\mathrm{sp}^{3}\right)$ & $0.55 \pm 0.14$ & $0.74 \pm 0.04$ & $0.063 \pm 0.005$ & $0.13 \pm 0.01$ & - \\
\hline Part of carbon $\mathrm{sp}^{2}, \%$ & $35 \pm 8$ & $42 \pm 1$ & $5.9 \pm 0.6$ & $12 \pm 2$ & 100 \\
\hline $\mathrm{F}_{0}$ & 16 & 52 & 0.52 & 1.1 & 548 \\
\hline
\end{tabular}

From the calculated data it is possible to make a series of increasing the ratio of the amount of surface carbon in $\mathrm{sp}^{2}$-hybridization to the amount of surface carbon in $\mathrm{sp}^{3}$-hybridization:

$\mathrm{ND}-\mathrm{CD}\left(\mathrm{HClO}_{4}\right)<\mathrm{ND}-\mathrm{CD}\left(\mathrm{HNO}_{3}+\mathrm{H}_{2} \mathrm{SO}_{4}\right)<\mathrm{ND}<\mathrm{CDDND}<\mathrm{CD}$

\section{PHOTOLUMINESCENCE SPECTROSCOPY OF AQUEOUS SUSPENSIONS OF CNP}

Photoluminescence spectra of the prepared aqueous suspensions of all samples of CNP were obtained when excited by laser radiation with a wavelength of $405 \mathrm{~nm}$. The concentration of all samples, except CD, was 1 $\mathrm{g} / \mathrm{l}, \mathrm{CD}$ concentration was $0.001 \mathrm{~g} / \mathrm{l}$. The value of $\mathrm{pH}$ for all suspensions was 8 . The obtained photoluminescence spectra of CNP under $405 \mathrm{~nm}$ excitation are shown in Figure 3. The band with maximum near $470 \mathrm{~nm}$ is the band of valence vibrations of $\mathrm{OH}$ groups of water.

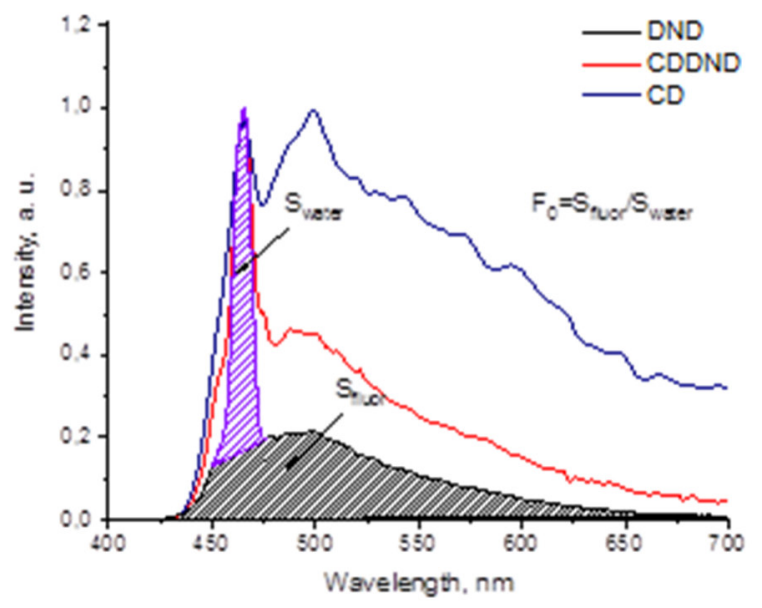

Figure 3 Photoluminescence spectra of aqueous suspensions of CNP excited by laser radiation with wavelength of $405 \mathrm{~nm}$. ( $\mathrm{pH}=8)$. Illustration of calculation of parameter $\mathrm{F}_{0}$

In order to quantitatively characterize the photoluminescence of samples, the parameter $\mathrm{F}_{0}$ was calculated. It is equal to the ratio of the integral photoluminescence intensity of CNP Sfluor to the integral intensity of the water Raman valence band Swater (Figure 3) [18]:

$F_{0}=S_{\text {Fluor }} / S_{\text {Water }}$,

where:

Sfluor - integral intensity of fluorescence

$S_{\text {water }}$ - integral intensity of water Raman valence band 
Parameters $F_{0}$ were calculated for all obtained photoluminescence spectra of aqueous suspensions. For suspensions of CD this parameter was recalculated for the CD concentration in water $1 \mathrm{~g} / \mathrm{L}$. The values of parameters $F_{0}$ are presented in Table 1. It should be noted that acid treatment of CDDND samples led to significant weakening of their photoluminescent properties: the parameters $\mathrm{F}_{0}$ for $\operatorname{CDDND}\left(\mathrm{HClO}_{4}\right)$ and $\mathrm{CDDND}\left(\mathrm{H}_{2} \mathrm{SO}_{4}+\mathrm{HNO}_{3}\right)$ are extremely small.

It follows from the results that there is an unambiguous relationship between the amount of carbon of the nondiamond phase on the surface and the intensity of the photoluminescence of the CNP: greater amount of nondiamond carbon on the surface corresponds to more intense photoluminescence of the samples. On the basis of a comparative analysis of the calculated parameters $F_{0}$, the following sequence of CNP according to the intensity of their photoluminescent properties (in ascending order):

$\mathrm{ND}-\mathrm{CD}\left(\mathrm{HClO}_{4}\right)<\mathrm{ND}-\mathrm{CD}\left(\mathrm{HNO}_{3}+\mathrm{H}_{2} \mathrm{SO}_{4}\right)<\mathrm{ND}<\mathrm{CDDND}<\mathrm{CD}$

The obtained sequence corresponds well with series (1) of increasing ratio of surface carbon in $\mathrm{sp}^{2}$ hybridization to surface carbon in $\mathrm{sp}^{3}$ hybridization. This agreement once again confirms the hypothesis that the more carbon in the non-diamond phase there is on the surface of CNP, the more intense is photoluminescence of these nanoparticles.

\section{CONCLUSION}

Photoluminescence of CNP with different amounts of carbon in the non-diamond phase on the surface of nanoparticles was experimentally investigated. The results of confocal Raman spectroscopy of ND, CD, ND$\mathrm{CD}, \mathrm{CDDND}\left(\mathrm{HClO}_{4}\right), \mathrm{CDDND}\left(\mathrm{HNO}_{3}+\mathrm{H}_{2} \mathrm{SO}_{4}\right)$ and photoluminescence spectroscopy of aqueous suspensions of these nanoparticles indicate that the intensity of the photoluminescence of CNP in water uniquely depends on the ratio of surface carbon in the non-diamond phase and surface carbon with $\mathrm{sp}^{3}$ hybridization. Greater amount of carbon in the non-diamond phase on the surface of CNP corresponds to more intense photoluminescence of these nanoparticles.

This study has been performed at the expense of the grant of Russian Science Foundation project No 17-1201481.

\section{REFERENCES}

[1] FAN J. and CHU P. Group IV Nanoparticles: Synthesis, properties, and biological applications. Small. 2010, 6 (19), 2080-2098.

[2] SCHRAND A., HENS S. C. and SHENDEROVA O. Nanodiamond Particles: Properties and Perspectives for Bioapplications. Critical Reviews in Solid State and Materials Sciences. 2009, 34(1), 18-74.

[3] ROSENHOLM J. M., VLASOV I. I., BURIKOV S. A., DOLENKO T.A. and SHENDEROVA O.A. NanodiamondBased Composite Structures for Biomedical Imaging and Drug Delivery (Review). J. of Nanoscience and Nanotechnology, 2015, v.15, pp.959-971

[4] KRUEGER A. New carbon materials: Biological applications of functionalized nanodiamond materials. Chem. Eur. J. 2008, 14, 1382-1390.

[5] DOLENKO T. A., BURIKOV S.A., LAPTINSKIY K.A., LAPTINSKAYA T.V., ROSENHOLM J.M., SHIRYAEV A.A., SABIROV A.R. and VLASOV I.I. Study of adsorption properties of functionalized nanodiamonds in aqueous solutions of metal salts using optical spectroscopy. J. of Alloys and Compounds, 2014, v.586, pp. S436-S439

[6] PRABHAKAR N., NAREOJA T., VON HAARTMAN E., KARAMAN D., JIANG H., KOHO S., DOLENKO T., HANNINEN P., VLASOV D., RALCHENKO V., HOSOMIU S., VLASOV I., SAHLGREN C. and ROSENHOLM J. Core-shell designs of photoluminescent nanodiamonds with porous silica coatings for bioimaging and drug delivery II: Application. Nanoscale, 2013, 5(9), 3713-3722. 
[7] VON HAARTMAN E., JIANG H., KHOMICH H., ZHANG S., BURIKOV S. A., DOLENKO T. A., RUOKOLAINEN T, GU H., SHENDEROVA O., VLASOV I. and ROSENHOLM J. Core-shell designs of photoluminescent nanodiamonds with porous silica coatings for bioimaging and drug delivery I: Fabrication. J. of Materials Chemistry B. 2013, 1(18), pp.2358 - 2366

[8] ROBERTSON J. and O'REILLY E.P. Electronic and atomic structure of amorphous carbon // Physical Review B. 1987. T. 35. № 6. C. 2946-2957.

[9] YAN J.-A., XIAN L. and CHOU M.Y. Structural and Electronic Properties of Oxidized Graphene // Physical Review Letters. 2009. T. 103. № 8

[10] CHIEN C.-T. Tunable Photoluminescence from Graphene Oxide // Angewandte Chemie International Edition. 2012. T. 51. № 27. C. 6662-6666Yan J.-A., Xian L., Chou M.Y. Structural and Electronic Properties of Oxidized Graphene // Physical Review Letters. 2009. T. 103. № 8

[11] FIELD J. The Properties of Natural and Synthetic Diamond. 1992. C. 710

[12] SHENDEROVA O., HENS S., VLASOV I., TURNER S., LU Y. G., VAN TENDELOO G., SCHRAND S., BURIKOV S. A. and DOLENKO T. A. Carbon-Dot-Decorated Nanodiamonds, Part. Part. Syst. Charact. 2014, https://doi.org/10.1002/ppsc.201300251

[13] DOLENKO T.A.. Optical imaging of fluorescent carbon biomarkers using artificial neural networks // Journal of Biomedical Optics. 2014. T. 19. № 11. C. 117007

[14] PETIT T. and PUSKAR L. FTIR spectroscopy of nanodiamonds: Methods and interpretation. Diamond and Related Materials, 2018, 89, 52-66. https://doi.org/10.1016/j.diamond.2018.08.005

[15] AGER J.W., VEIRS D.K. and ROSENBLATT G.M., Phys. Rev. B 43 (1991) 6491

[16] OSSWALD S.; MOCHALIN, V. N.; HAVEL YUSHIN, G. and GOGOTSI Y. Phonon Confinement Effects in the Raman Spectrum of Nanodiamond. Phys. Rev. B 2009, 80, 075419. https://doi.org/10.1103/PhysRevB.80.075419

[17] LE K. C., LEFUMEUX C. and PINO T. Differential Raman backscattering cross sections of black carbon nanoparticles. Scientific Reports, 2017, v.7, p.17124

[18] DOLENKO T.A., BURIKOV S.A., LAPTINSKIY K.A., ROSENHOLM J.M., SHENDEROVA O.A. and VLASOV I.I. Evidence of carbon nanoparticle - solvent molecule interactions in Raman and fluorescence spectra. Physica Status Solidi A, 2015, v.212, N11, pp.2512-2518 